\title{
ESTADO Y CAPITAL SOCIAL EN AMÉRICA LATINA: ¿EN QUÉ MEDIDA LAS CARACTERÍSTICAS Y COMPORTAMIENTOS DEL ESTADO EXPLICAN LOS NIVELES DE CAPITAL SOCIAL EN LA REGIÓN? \\ State and social capital in Latin America: In which way the features \\ and actions of the State explain the social capital levels in \\ Latin America?
}

\author{
María Cecilia GÜEMES \\ Universidad Nacional del Litoral, Argentina \\ $\triangle$ cecilia.guemes@gmail.com
}

BIBLID [1130-2887 (2011) 59, 91-116]

Fecha de recepción: 15 de octubre del 2009

Fecha de aceptación y versión final: 12 de mayo del 2011

RESUMEN: El presente trabajo se propone indagar en qué medida el Estado y las políticas públicas son capaces de potenciar la creación del capital social. Tras reseñar la importancia del capital social para el desarrollo, los debates en torno a su definición, sus debilidades y sus promesas, la primera parte de este artículo se centra en su problemática reproducción, prestando especial atención al papel del Estado y las políticas públicas como catalizadores o promotores del mismo. En vista a ello, la segunda parte del trabajo propone una modesta aproximación empírica a la correlación entre Estado y capital social en el contexto de América Latina. Se utilizan al efecto indicadores de desempeño/eficacia estatal, otros asociados al bienestar social (principalmente gasto público y desigualdad) y percepciones ciudadanas en referencia a la confianza interpersonal. Se concluye retomando los resultados del abordaje empírico y proponiendo futuras líneas de problematización en la materia.

Palabras clave: capital social, desarrollo, Estado, políticas públicas, América Latina.

ABSTRACT: By focusing on the importance of social capital to development, this paper examines the role of the State and Public Policy in the creation of social capital. After a brief summary about social capital definitions, weakness and usefulness, its relevance to development 
and its problematic creation, the paper pays specific attention to the role of the State as a promoter of social capital. Second we bear out the empiric links between State performance and social capital in Latin America. Then, we use state's efficacy indicators, variables traditionally associated with welfare stat (especially public spending and inequality) and interpersonal trust. We finish by pointing the previous findings and shedding light in order to continue the research about social capital reproduction.

Key words: social capital, development, State, public policy, Latin America.

\section{INTRODUCCIÓN ${ }^{1}$}

Ríos de tinta se han derramado desde que Putnam (1993) aventurara una correlación positiva entre capital social, desempeño gubernamental, desarrollo y democracia. Pese a la poca claridad de la idea y su problemática medición, resultados exitosos en materia de crecimiento, productividad, innovación, competitividad y bienestar han sido asociados y explicados a partir de altos niveles de capital social o, más precisamente, a indicadores de confianza social, asociacionismo o cooperación informal (Granovetter 1985; Evans 1996b; Knack y Keefer 1997; Woolcock y Narayan 2000; Puntam 2001; Bowles y Gintis 2001; Ostrom y Ahn 2003; Dasgupta 2003; Trigilia 2003; Bagnasco 2005; Caballero y Kingston 2005; Olazaran et al. 2005; Rodríguez y Román 2005; Sánchez y Pena 2005).

Tomando en consideración escenarios como el latinoamericano, donde el desarrollo es uno de los objetivos más urgentes y a su vez los «stocks» de capital social parecen ser relativamente bajos, el interrogante que se impone es: ¿Qué vías o caminos existen para fomentar la emergencia del capital social?

Adentrarse en tal consigna supone previamente pasar revista a los debates en torno a la conceptualización y caracterización del capital social, las limitaciones y ambigüedades del enfoque y su relevancia para desarrollo. Hecho esto, en lo que resta de la primera parte de este artículo, se puntualizarán las principales cuestiones que rodean la problemática creación del mismo, prestando especial atención a las líneas de investigación que aventuran al Estado y las políticas públicas como agentes claves en la potenciación del mismo.

Tomando como referencia los aportes que se derivan de la parte II, en la parte III del trabajo se busca aproximarse a la vinculación entre ciertos factores asociados al desempeño del Estado y al capital social en el contexto latinoamericano. De este modo, se intenta visualizar si la competitividad y eficacia estatal, el gasto social y ciertos indicadores

1. El presente trabajo forma parte de la investigación doctoral de la autora en el Instituto Ortega y Gasset y la Universidad Complutense de Madrid y ha sido posible gracias al apoyo del Programa Alban, Becas de Alto Nivel de la Unión Europea para América Latina, beca n. ${ }^{\circ}$ E07D402401AR. Se agradece a Francisco Herreros Vázquez su inestimable ayuda, apoyo y comentarios a lo largo de la elaboración del presente trabajo, así como las observaciones y comentarios formulados por los revisores anónimos de la revista América Latina Hoy, Revista de Ciencias Sociales. 
tradicionalmente asociados al bienestar ciudadano contribuyen a explicar los niveles diferenciales del capital social en América Latina. Atento a la simplicidad del aparato estadístico que se utilizará, el análisis que se obtiene representa un primer acercamiento a la comprobación de la hipótesis y sirve para conocer el estado de la situación en la región. Los resultados encontrados, si bien no son suficientes para afirmar una relación de causalidad, prueban cuando menos que dicha relación no debe descartarse y antes bien explorarse en profundidad.

Se concluye el trabajo retomando las principales ideas teóricas presentadas y los resultados derivados del abordaje empírico a la par que se ofrecen algunas pistas útiles para futuras indagaciones en la materia.

\section{Claves teóRICAS}

\section{II.1. Concepto, características y tipos de capital social. Debilidades y promesas del enfoque}

La perspectiva del capital social viene a poner el acento sobre temas tales como: el mundo de las relaciones cara a cara, las redes de cooperación, el asociacionismo, las normas formales e informales que moldean y coordinan las acciones sociales, la solidaridad y confianza social, entre otros (Putnam 1993; Jordana 2000; Torcal y Montero 2000; Herreros y De Francisco 2001).

Si tales cuestiones no son nuevas para la teoría sociológica, la psicología social o la ciencia política, pasaron sin embargo, durante un largo tiempo, desapercibidas o soslayadas para los teóricos del desarrollo económico. Por ello, el concepto de capital social resultó bienvenido, constituyéndose en un llamado de atención dirigido a los teóricos para que salgan del mundo del razonamiento puro, aborden cuestiones de importancia práctica que atraviesan distintos campos de investigación y formulen nuevos instrumentos para el análisis empírico, así como un esfuerzo por relativizar las relaciones causales tradicionales que dan prioridad a la explicación económica por sobre la social o las argumentaciones netamente micromotivacionales o macroestructurales (Ostrom y Ahn 2003; Jordana 2000; Levi 1996).

Pese a la ambigüedad del concepto, ajustable a todos los gustos y corrientes ideológicas, pueden diferenciarse dos grandes líneas de teorización: la «culturalista» (centrada en actitudes y valores) y la «estructuralista» (enfocada en el papel de la historia y de las estructuras).

En el marco del enfoque «actitudinal» o «culturalista», el fenómeno del capital social se concibe como algo subjetivo asociado a ciertos rasgos individuales y relacionado con preferencias, valores y actitudes personales construidos al abrigo de una cultura y memoria social histórica específica (Herreros 2002). Pese a sus notorias diferencias y bagajes ideológicos, tales estudios se enmarcan en la corriente sociopolítica inaugurada por los trabajos de Weber (1905), Lipset (1959) y Almond y Verba (1970) destacando el papel de la cultura cívica en la estabilidad y calidad democrática, como en el desarrollo y la 
prosperidad económica ${ }^{2}$. A título de ejemplo pueden verse los trabajos de Putnam (1993, 2001), Inglehart (1998) o Torcal y Montero (2001).

Atento a las dificultades que plantea identificar las raíces históricas en las que se sustenta la cultura actual de una sociedad, así como los principales factores involucrados con el cambio cultural, en el caso de regiones no bendecidas (donde los círculos viciosos entre capital social, democracia y desarrollo están a la orden del día), explicaciones de este tipo ofrecen un pronóstico muy desalentador, pues la suerte de tales regiones parece haberse definido largo tiempo atrás (Trigilia 2003; Güemes et al. 2006).

Respecto al enfoque «estructuralista», el mismo se halla fundado en torno a los desarrollos de Bourdieu (2001), Coleman (2001) y Granovetter (1985). Para estos autores, el capital social radica en un aspecto de la estructura social que permite a los sujetos acceder a redes e interrelaciones, pudiendo explicarse la cooperación como una opción racional inducida o influida por ciertos incentivos o sanciones estructurales antes que como la expresión de una pauta cultural interiorizada (Boix y Posner 2000; Jordana 2000; Herreros 2002).

Dentro de este último enfoque pueden distinguirse dos vertientes teóricas diferentes: a) una de inspiración marxista, claramente expuesta por Bourdieu, que entiende el capital social como privilegio de clase y por tanto lo analiza como un camino por el que se reproducen y refuerzan las desigualdades y el dominio de clase y b) una lectura de matriz más durkheimiana, donde el capital social es visualizado como un bien público que consiste en ciertos rasgos socioestructurales o recursos que son accesibles a los individuos y grupos a partir de las redes que estos construyen. Exponentes de esta línea son los trabajos de Coleman (2001) y Lin (1999, 2000).

Revalorizando las estructuras sociales y el entorno institucional, político, económico y social como marco de acción y relación social, este enfoque parece ofrecer un mayor margen de acción para que, instrumentos como la política pública reviertan legados negativos e incentiven la creación de capital social (Levi 1992).

En cuanto a la configuración de tipos o categorías de capital social, tomando como referencia la precursora diferenciación de Granovetter (1973) entre lazos débiles (de largo alcance) y fuertes (asociados a las redes familiares o de amistad), se ha resaltado la importancia para el desarrollo y el desempeño gubernamental de los lazos no ya intrafamiliares o intraétnicos sino entre «extraños».

Claramente, el tipo de lazo que importa dependerá del contexto en el que se problematiza el capital social. Así por ejemplo si se piensa en la movilidad de trabajos calificados en grandes ciudades, es probable que el lazo débil sea más relevante, en cambio, si se investigan los lazos entre inmigrantes en ciudades industriales o suburbios, quizá sean más decisivos los lazos fuertes en tanto apoyo y contención (Lin 1999; De la Maza 2001; Pisseli 2003; Ayerbe et al. 2005). Sin embargo, de cara a los desafíos actuales, la mayoría de los estudios prefieren centrarse en las relaciones interpersonales abiertas

2. Si bien el posicionamiento de Putnam se acomoda muy bien a los enfoques de la cultura política, también es cierto que incorpora características propias del enfoque estructural como las redes sociales (F. HERRERos y H. DE CRIADO 2001: 8). 
y en su potencial para favorecer salidas o soluciones cooperativas y coordinadas a los diversos asuntos que los actores sociales van enfrentando (Granovetter 1973; Knack 2000; Woolcock y Narrayan 2000; Durston 2000 y 2003; Boix y Posner 2000; Sabatini 2005).

Por su parte, en lo que respecta a las virtudes del enfoque del capital social, se han señalado múltiples beneficios en diferentes niveles: «individual» (sentimiento de felicidad, satisfacción con la vida, optimismo a futuro o sentimientos de utilidad personal), «social» o «cultural» (cohesión grupal, respeto, tolerancia y valoración grupal, mejora en las relaciones y vínculos sociales, etc.), «político» (mayor participación y diálogo, fortalecimiento del civismo, mayor control ciudadano, etc.) y «económico» (facilita la actividad productiva y contribuye al desarrollo económico y social de las sociedades).

Frente a tal optimismo cabe ahora mencionar los trabajos que señalan las debilidades y lados oscuros del enfoque. Para empezar, suelen citarse una profusa ambigüedad conceptual y amplios desacuerdos en torno a su definición, la identificación de sus componentes y sus formas; todo lo cual dificulta el intercambio y debate académico y da lugar a la proliferación de estudios que aventuran el capital social como variable explicativa de casi cualquier cosa o panacea resolutiva de todos los males (Lin 1999; Woolcock y Narrayan 2000; Knack 2000; De la Maza 2001; Durlauf 2002; Sánchez y Pena 2005; Bebbington 2005).

La imposibilidad de distinguir con claridad entre aquello que causa el capital social y lo que resulta de él conduce, las más de las veces, a aproximaciones tautológicas o circulares de las variables en estudio. Deriva de lo anterior un fuerte sesgo determinista o hiperfuncionalista que subvalora el papel de los actores en el cambio, la dinámica de la cultura y el papel de las variables políticas (Portes 1998; Boix y Posner 2000; Herrero y De Francisco 2001; Piselli 2003; Bebbington 2005; Bagnasco 2003). Asimismo, también se ha señalado que no todo capital social es valioso o positivo socialmente ni tampoco siempre fungible, intercambiable o trasladable desde un espacio a otro (Portes 1998; Durston 2000; Levi 2001; Bowles y Gintis 2001; Piselli 2003; Katzman 2003).

Sumado y derivado de los dos puntos anteriores se verifica una escasa atención a la articulación entre el capital social y las desigualdades de poder, las diferencias de género, el clientelismo, la exclusión de extraños, las restricciones a la libertad individual, entre otros (Portes y Ladolt 2000; Levi 2001; Caballero y Kingston 2005; Ayerbe et al. 2005; CEPAL, 2002). Sin perder de vista y compartiendo muchas de estas críticas generales, se cree no obstante que el enfoque del capital social puede ser útil para reflexionar el desarrollo por las siguientes razones:

a. En sentido general, dicha categoría permite re-oxigenar la problematización del desarrollo atento a que revaloriza problemáticas sociales que subyacen al desarrollo económico y bienestar social, recoloca el foco de atención no tanto en la acción individual sino en las relaciones sociales y hace dialogar lo macroestructural con lo micromotivacional.

b. En términos más concretos, se puede afirmar que el capital social aporta al desarrollo económico en varios niveles.

b.1. A nivel micro- y mesorregional se suele señalar que la mayor comunicación y confianza a que dan lugar los lazos interpersonales reduce los 
costos de transacción haciendo posibles formas de organización que garantizan mayor seguridad entre los participantes. De este modo, se ahorran recursos destinados tanto a la defensa de acciones desleales o delictivas como a la negociación y ejecución de contratos, los que pueden luego emplearse en inversiones destinadas a la innovación, transferencia de tecnología e investigación de procesos y productos, al desarrollo de créditos informales o la formación de capital humano, etc.

Juntos, mayor comunicación entre los actores reduce riesgos de oportunismo y mejora la trasmisión y socialización de conocimientos e información (sea ésta sobre cuestiones sustantivas como sobre preferencias de los miembros de la red social). Todo ello aporta certeza y amplía los horizontes temporales facilitando la coordinación de acciones y la adopción de elecciones colectivas en contextos de incertidumbre y asimetría informativa (Coleman 2001; Lin 1999; Herreros 2002; Knack y Keefer 1997; Boix y Posner 2000; Jordana 2000; Díaz Albertini 2001; Bagnasco 2003; Cornejo 2005; Caballero y Kingston 2005; Ayerbe et al. 2005; Olazarán et al. 2005; Rodríguez Modroño y Román del Río 2005).

b.2. Derivado de los efectos a nivel interpersonal o microsocial se concluye a nivel macro que el capital social fortalece la gobernabilidad democrática, hace más eficiente la administración pública y eleva la calidad de las políticas económicas (al mejorar los canales y vías de comunicación sinérgica entre el Estado y la sociedad civil) contribuyendo por tanto al desarrollo económico de una región (Putnam 1993; Evans 1996; Knack y Keefer 1997).

Atento a estas promesas los interrogantes abiertos son: ¿Qué esperanza cabe a las regiones poco dotadas de capital social o con energía social adormecida o latente, en clave de Hirschman (1986), para desarrollar nuevos patrones de correlación e interrelación social? ¿Es posible una visión del capital social «posibilista» o constructivista? ¿Pueden el Estado y las políticas públicas promover o favorecer la emergencia del capital social? Sobre estas preguntas se trata en el acápite que sigue.

\section{II.2. Reproducción y creación del capital social. Introducir al Estado y la política en el debate}

Adentrarse en la reproducción/creación del capital social supone enfrentarse a varios problemas. En primer término, como es común en las ciencias sociales, resulta sumamente difícil establecer relaciones de causalidad claras entre capital social y variables como la democracia, el desarrollo o el desempeño estatal. En segundo término, su oferta conjunta e imposibilidad de exclusión asemeja el capital social a los bienes públicos ${ }^{3}$,

3. Dicha afirmación se sustenta en que, el capital social, requiere de una inversión social de tiempo y esfuerzo; no es un bien que pueda pertenecer en propiedad a un solo sujeto, sino mas bien a los miembros de un colectivo (lo cual no obsta a que ciertos grupos o sujetos puedan controlar el acceso 
lo que enfrenta a las dificultades propias de la acción colectiva: faltas de incentivos para su producción, el problema de penalizar a los oportunistas, el dilema del prisionero y los problemas de coordinación ${ }^{4}$.

En vista a ello, las soluciones propuestas suponen alentar ciertas actividades para que el capital social surja como subproducto de las mismas o intervenir sobre ciertas estructuras que precondicionan su creación. A continuación se sintetizan algunas propuestas tomando como referencia el enfoque privilegiado en la conceptualización del fenómeno:

b.1. Opciones culturalistas: focalizan en el poder de las políticas educativas y asociativas como espacio de promoción e interiorización de valores sociales y comunitarios.

b.2. Opciones institucionalistas y estructuralistas: proponen cambiar reglas y estructuras en las cuales se insertan las acciones y relaciones sociales.

En lo que respecta a las opciones culturalistas, centradas en la educación en valores colectivos y republicanos o el refuerzo de códigos éticos y morales, tienen a los estudios de cultura cívica y política como un mojón histórico del cual asirse (Almond y Verba 1970). Así, dado que la cultura cívica se desarrolla en cada individuo a partir de experiencias socializadoras dependientes de prácticas tempranas (propias del núcleo familiar) y posteriores (desarrolladas en los ambientes educativos y en menor medida laboral) que permiten que estos principios se incorporen en el marco axiológico y cognitivo de los sujetos, la promoción de valores debe acompañarse de medidas objetivas que incrementen la frecuencia y la calidad de las oportunidades de sociabilidad informal en condiciones de igualdad con miembros de otras clases sociales (Katzman 2003, 2007). Sobre este asunto poco se ha escrito hasta el momento en lo que refiere a capital social.

Mayor relevancia ha asumido el fomento del asociacionismo o trabajo voluntario como herramientas de mejora y fortalecimiento de la solidaridad social (Putnam 1993, 2001). Dicha propuesta se sustenta en los clásicos trabajos de Tocqueville o Durkheim, suponiendo que la interacción repetida entre sujetos es capaz de incrementar las redes sociales del individuo y desarrollar una mayor predisposición a la acción colectiva. Ahora bien: ¿Es la participación en asociaciones la que genera confianza o más bien la confianza lo que motoriza la participación? ¿La confianza particularizada que surge en el marco de las asociaciones y el voluntariado es extrapolable a sujetos ajenos o diferentes? ¿Cuánto tiempo deben los sujetos permanecer en una asociación para que se desarrolle confianza? ¿Da lo mismo si la asociación está vertical u horizontalmente organizada,

o aprovecharse diferencialmente de él); una vez creado puede beneficiar a todos los que pertenecen a la estructura social en cuestión (F. Aguiar 1990; J. Coleman 2001; E. Ostrom 2003; J. SÁnChez y J. PENA 2005).

4. Recordar que las redes sociales no son meros bienes de inversión pues a menudo crean valor de consumo directo y su producción comporta en múltiples casos un placer en sí mismo para quienes lo desarrollan (R. Putnam y K. Goss 2002; A. HiRschman 1986b). 
se financia con recursos estatales o privados, es formal o informal, homogénea o heterogénea? De acuerdo a dichos interrogantes, una serie de trabajos cuestionan la romántica y mítica imagen de las asociaciones remarcando como, en sociedades polarizadas, el asociacionismo puede derivar en efectos perversos facilitando el rent seeking, haciendo lobby respecto a sus propios intereses o entrando en conflicto con otros intereses sociales organizados o la sociedad en general (Knack y Keefer 1997; Stolle 2000; Boix y Posner 2000; Mota 2000; Herreros y De Francisco 2001; De la Maza 2001; Sabatini 2005; Lechner 2000; Sánchez y Pena 2005).

Por último, las estrategias institucionalistas y estructuralistas (b.2) apuestan a la transformación de las bases de interacción actuando a nivel de las oportunidades de los sujetos (Elster 2003). Resalta al efecto el papel de las instituciones formales e informales como fuerzas capaces de: a) modelar comportamientos individuales mediante reglas, castigos e incentivos y b) solucionar problemas de acción colectiva y reducir los fallos de coordinación espontánea mediante la asignación de recursos e información, sea esto: mediante mejoras institucionales tendientes a asegurar el cumplimiento de contratos, la regulación de las relaciones económicas o la formalización de códigos éticos (Knack y Keefer 1997; Knack 2000; Jordana 2000)5.

La ventaja de esta última posición es que re-contextualiza el problema de las relaciones sociales en el marco de las estructuras e instituciones en que se desarrollan las mismas, cobrando importancia las políticas públicas que dan forma y construyen tales estructuras e instituciones y el Estado como actor de relevancia en la creación y promoción del capital social.

Así, mientras algunos autores advierten del peligro de que el Estado destruya al capital social, otros entienden que su acción puede ser eficaz al momento de revertir estructuras y rutinas sociales signadas por dilemas de acción colectiva de modo de favorecer un clima «amigable» con confianza social. La argumentación procede del siguiente modo. Si el Estado es el proveedor último de los bienes públicos, el árbitro final de controversias y el responsable del Estado de Derecho, quizá sea el sujeto que en mejores condiciones está para: a) facilitar y garantizar alianzas duraderas, b) favorecer la circulación de información reduciendo con ello los costos de transacción y problemas de coordinación, c) monitorear acuerdos y sancionar a los free riders y d) promover valores cooperativos así como instancias de socialización que, más allá de las diferencias de clase, etnia, género o políticas, favorezca la confianza interpersonal (Levi 1996; Portes 1998; Tarrow 1996; Scholz y Gray 1997; Torcal y Montero 2000; Boix y Posner 2000; Herreros 2002; Knack 2000; Woolcock y Narrayan 2000; Putnam 2001).

Invirtiendo la tradicional relación que ve al capital social como causa del buen gobierno, en lo que sigue se pretende analizar si existe una relación positiva entre Estado y capital social. Específicamente, en qué medida el desempeño del Estado y ciertas opciones políticas pueden favorecer dicho capital.

5. Sin embargo, debe tenerse en cuenta que las instituciones no siempre son capaces de determinar los comportamientos o configurar la cultura (M. TORCAL y J. R. MONTERO 2000; J. SiDES 1999) y, en ciertos casos, pueden manipularse en beneficio de algunos pocos (M. TAYLOR 2001; P. BOURDIEU 2001). 
Como primera hipótesis se sugiere que Estados con eficaces estructuras se correlacionan con altos niveles de capital social y, a la inversa, Estados débiles o incapaces se acompañarían de bajos niveles de capital social. El fundamento de tal afirmación radicaría en que estructuras estatales eficaces contribuyen a que el funcionamiento del mercado sea más trasparente, los sistemas de arbitraje más sencillos y rápidos, las conductas oportunistas sancionadas, los acuerdos que ponen en peligro la acción colectiva supervisados, las acciones cooperativas incentivadas y la información coordinada y distribuida. Atento a ello, Estados eficaces serían forjadores de un escenario favorable a la emergencia de la confianza y el capital social (Knack y Keefer 1997; Herreros 2007; Bowles y Gintis 2001; Ostrom y Ahn 2003). En segundo término, otro probable camino por el que el buen desempeño de las instituciones estatales favorece la emergencia de capital social sería que la mayor eficiencia del Estado aumenta la confianza de la ciudadanía en dichas instituciones e, indirectamente, este tipo de confianza institucional repercute y favorece la confianza interpersonal (Newman-Norris 2000; Rothstein 2002, 2005; Herreros 2007).

Como segunda hipótesis se sostiene que no sólo la eficiencia del aparato es importante en la recreación del capital social, sino también el tipo de políticas públicas que promueve y la sociedad que construye dicho Estado. En este sentido, más allá de políticas que específicamente se puedan proponer construir capital social, el Estado puede incentivar el capital social influyendo sobre variables que íntima y causalmente están asociadas a la promoción de la igualdad económica y de oportunidades. Dicha hipótesis se apoyaría en:

a. la comprobada correlación negativa que existe entre la confianza interpersonal y la desigualdad económica y de oportunidades (Knack y Keefer 1997; Kawachi et al. 1997; Sides 1999; Hall 2002);

b. una correlación positiva hallada en análisis comparados europeos entre confianza interpersonal y ciertos modelos de Estado de Bienestar (Rothstein 2002, 2008; Rothstein y Uslaner 2005; Herreros 2008).

De lo anterior se deduce que un involucramiento activo del Estado en la provisión de políticas aseguradoras del bienestar colectivo es fundamental para evitar la polarización social, construir una idea de comunidad, sentidos compartidos y destino común, lo que fomenta lazos de cohesión y confianza social (Boix y Posner 2000; Jordana 2000; Lechner 2000; Bowles y Gintis 2001; Ostrom y Ahn 2003)6.

6. En el marco de las políticas tendientes a la igualación de oportunidades especial atención merecen las políticas de cobertura universal atento a que poseen: 1) mayor potencial redistributivo en comparación con programas focalizados, 2) mayor eficiencia para promover la igualdad económica y tratamientos más equitativos y menos propicios a la discrecionalidad y clientelismo, 3) menores costos administrativos y burocráticos, 4) potencial para construir a nivel simbólico un sentimiento de solidaridad social y una percepción de destino común que reduce la segmentación y estigmatización propia de las políticas focalizadas (B. ROTHSTEIn 2002 y 2008; B. Rothstein y E. UsLanER 2005; R. MuÑOZ DE Bustillo 2005; P. GÜELl 2002). 
GRÁFICO I: VARIABLES E HIPÓTESIS BAJO ANÁLISIS

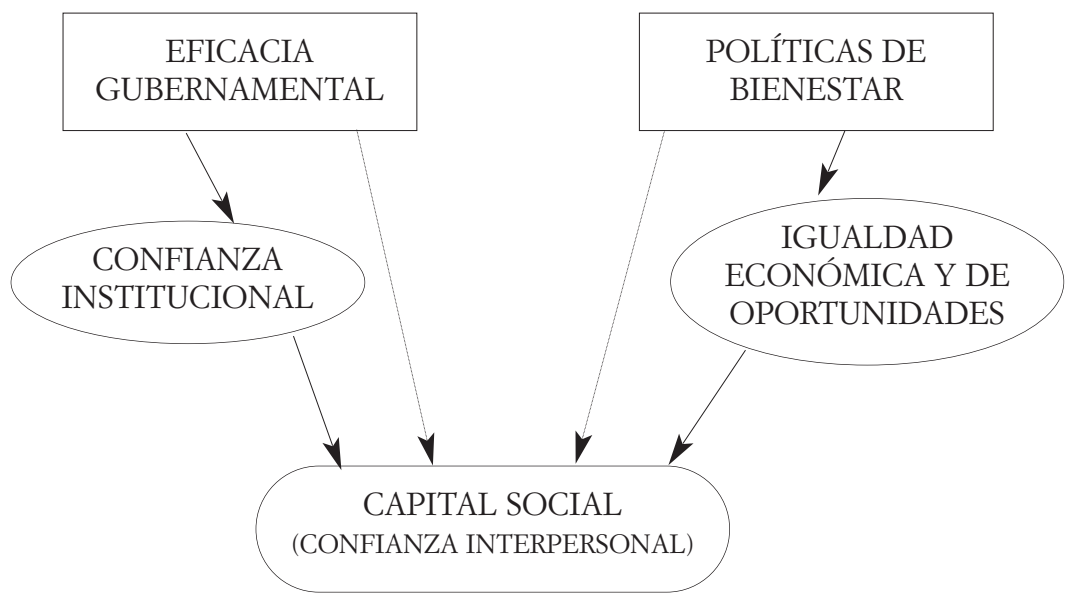

Fuente: Elaboración propia.

Tomando en consideración las hipótesis anteriores y los resultados positivos que hipótesis similares han encontrado en abordajes empíricos europeos, se debería poder comprobar en América Latina una correlación positiva entre el buen desempeño estatal, la confianza institucional y los niveles de capital social, por una parte, y las políticas de bienestar, equidad social y niveles de confianza social, por la otra. En los párrafos que siguen se ofrece una serie de correlaciones tendientes a verificar ambas cuestiones. Como se comentó anteriormente, este análisis exploratorio y descriptivo no alcanza para validar las hipótesis sino que constituye un primer ejercicio útil para introducirse en el estudio de la temática?.

\section{CLAVES EMPÍRICAS}

\section{III.1. Aclaraciones metodológicas}

Con el objetivo de sopesar la relación existente entre capital social y Estado en este apartado se utilizará una metodología estadística bivariable de correlaciones simples (Coeficiente $r$ de correlación de Pearson). Dicho abordaje es sincrónico y extensivo y se focaliza en la región latinoamericana, particularmente en: Argentina, Bolivia, Brasil,

7. El presente trabajo es parte de la investigación doctoral de la autora. Como tal, constituye un primer acercamiento a la cuestión. En la tesis propiamente dicha se ponen a prueba modelos estadísticos más sofisticados como regresiones logísticas y análisis multinivel. Así mismo, se diseña una estrategia metodológica cuanti-cualitativa orientada a un estudio de caso (Argentina) que tiene por finalidad comprender y explicar los mecanismos en virtud de los cuales las políticas públicas intervienen en la creación y dinámica de la confianza social. Ver al respecto M. C. GÜEMES (2011). 
Chile, Colombia, Costa Rica, Ecuador, El Salvador, Guatemala, Honduras, México, Nicaragua, Panamá, Paraguay, Perú, República Dominicana, Uruguay y Venezuela.

Para medir el capital social (variable dependiente) el indicador que se utiliza es la confianza interpersonal. La selección de dicho indicador se funda en que, pese a las críticas que se le han formulado ${ }^{8}$, el mismo continúa siendo el indicador más empleado en los estudios comparados de capital social, habiendo probado ser consistente con la impresión popular de sujetos del territorio en análisis, así como la opinión de observadores externos y cierta evidencia anecdótica derivada de experimentos realizados (Knack 2000). Básicamente, se toma en cuenta el porcentaje de respuestas afirmativas a la pregunta ¿Se puede confiar en la mayoría de las personas? La base de datos utilizada es Latinobarómetro para el año 2005.

Para evaluar la primera variable explicativa: el desempeño gubernamental/eficacia estatal, se incluye el Îndice Competitividad Global (ICG) incluido en el Reporte de Competitividad Global del Foro Económico Mundial para los años 2007-2008. El ICG provee una mirada holística sobre factores que son críticos para la productividad y competitividad y que pueden agruparse en nueve pilares: 1) Instituciones, 2) Infraestructura, 3) Macroeconomía, 4) Salud y Educación primaria, 5) Educación superior y capacitación, 6) Eficiencia de mercado, 7) Adaptación tecnológica, 8) Sofisticación comercial y 9) Innovación. Con el objetivo de concentrarse en la medición de la eficacia

8. Sendas críticas se han formulado a la medición del capital social a partir de indicadores como la confianza interpersonal. En primer lugar no está claro si la confianza es un resultante, una forma o un componente del capital social, por tanto, resulta cuestionable el uso de la confianza como indicador del capital social. En segundo lugar se han señalado las limitaciones que tienen las encuestas de opinión para evaluar los niveles de capital social puesto que: a) las respuestas están condicionadas por el grado de disposición individual, el cual es dependiente de la posición particular que quien responde ocupa en la estructura social, b) las respuestas son fácilmente influenciables por las particulares coyunturas que experimenta en ese momento quien responde así como por los medios de comunicación, c) las encuestas sólo reflejan una toma fija y estática del capital social perdiendo de vista su radio de alcance, intensidad, distribución y utilización, no siendo por lo general los resultados agregados representativos de la sociedad, d) inexistencia de estudios cualitativos que ayuden a interpretar lo que puede querer decir la gente cuando responde a preguntas tales como: si se confía en las personas en general, y qué sucedería si se cambia la pregunta y se consulta por ejemplo: si nunca se es suficientemente precavido al tratar con la gente (KNACK 2000; SABATINI 2005; DíAZ AlBERTINi 2001; WutHNOW 2002). En vista a ello se ha sugerido utilizar otros indicadores como: asociacionismo formal (afiliación a asociaciones), cooperación y voluntariado (cantidad de horas destinadas a dicho esfuerzo, el tipo de proyectos preferidos, etc.); el empoderamiento y participación cívica y política (apoyo a campañas políticas o participación en elecciones, participación informal en marchas, manifestaciones, protestas o movimientos sociales); la atención y receptividad de los ciudadanos respecto a lo que sucede en el entorno social (frecuencia en la consulta a medios de comunicación) (OFFE y Fuchs 2002; Putnam 1993; Sides 1999; Hall 2002; Putnam y Goss 2002; Welzel et al. 2005). Iniciativas como las del BM han intentado en estudios de casos combinar estos indicadores en un agregado. El mayor problema que se encuentra al respecto es que los elementos que integran dicho indicador agregado poseen covariaciones dispares entre ellos y en términos generales si se ponderan todos estos elementos por igual la dinámica de tal indicador agregado no varía mucho respecto a la observada con la confianza interpersonal. Ver gráficos en Anexo. 
de las estructuras y el desempeño estatal se utiliza un subíndice incorporado dentro del ICG denominado «Requerimientos Básicos», el cual incluye los cuatro primeros pilares antes mencionados.

Para analizar la segunda variable explicativa: políticas de bienestar, se utilizarán una batería clásica de indicadores agregados vinculados a la igualdad económica y de oportunidades, como son: Coeficiente de Gini, Índice de Capacidades Básicas’, esperanza de vida al nacer, promedio de años de escolaridad, tasa de escolarización y porcentaje de población de 25 años y más con nivel educativo superior y universitario completo. Asimismo, se utiliza el indicador de gasto público para analizar el involucramiento del Estado en estas materias. Las bases de datos utilizadas provienen en su mayoría de estudios de la CEPAL, Banco Mundial, OIT, UNESCO y Latinobarómetro.

\section{III.2. El capital social en América Latina: presentación de los resultados obtenidos}

Como introducción al tema, se presenta una gráfica descriptiva de los niveles de capital social, en la cual pueden observarse comparadamente los niveles de desarrollo, calidad democrática y confianza interpersonal en los países de la región.

En materia de confianza interpersonal los niveles observados, comparados con los europeos o estadounidense, tienden a ser bajos (media para América Latina $=19)^{10}$ y muy heterogéneos (Desviación estándar de 7,1). Dicho lo cual, no es extraño encontrarse con países como Uruguay o Argentina con valores similares a los europeos a la par de países como Brasil, con niveles de confianza social parecidos a los de países africanos como Ghana o Ruanda. Asimismo, parece comprobarse la existencia de círculos virtuosos donde los comparativamente altos niveles de capital social se acompañan de altos niveles de calidad democrática y desarrollo (Argentina, Chile, México o Uruguay) en oposición a círculos viciosos, donde los bajos niveles de capital social se compadecen con bajos niveles de calidad democrática y desarrollo (Honduras, Nicaragua, Paraguay o Perú). La correlación entre el índice de desarrollo humano y la confianza interpersonal es de 0,4; mientras que la correlación entre el índice de calidad democrática y la confianza interpersonal es de 0,32 .

Atento a los bajos y heterogéneos niveles de capital social encontrado, se ve entonces si la primera hipótesis se verifica para América Latina. Para ello observaremos las correlaciones existentes entre: 1) confianza interpersonal y eficacia estatal, 2) eficacia estatal y confianza institucional gubernamental y 3) confianza institucional gubernamental y confianza interpersonal.

9. Elaborado por Social Watch este índice representa el promedio simple de tres indicadores: el porcentaje de niños que llegan a quinto grado de enseñanza primaria, sobrevivencia hasta los cinco años (con base en la mortalidad de menores de cinco años) y el porcentaje de partos asistidos por personal especializado. Para más información ver http://www.socialwatch.org.

10. Ver en Anexo comparación de niveles de confianza social de países de América Latina con otras regiones. 
GRÁFICO II

CAPITAL SOCIAL, DESARROLlo Y CALIDAD DEMOCRÁTICA EN AMÉRICA LATINA (AÑOS 2005-2007)

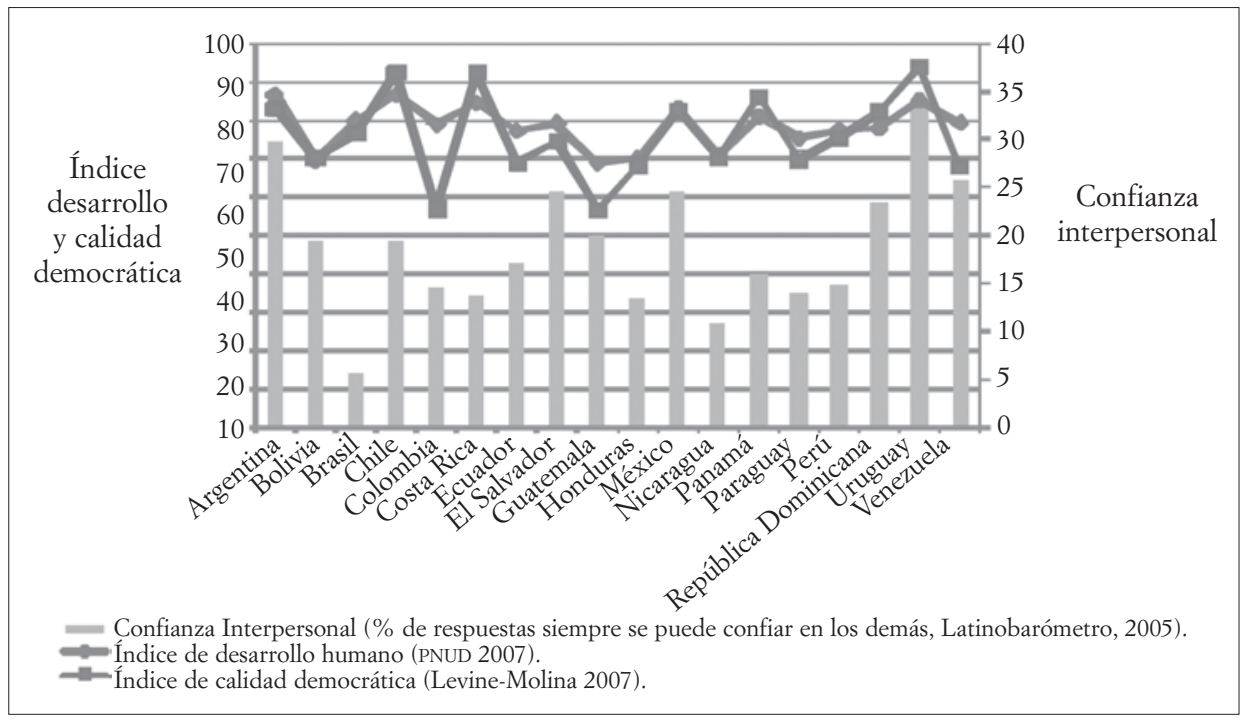

Fuente: Elaboración propia con base en datos de IDH, PNUD (2007); para calidad democrática D. LEVINE y J. Molina (2007) y para Confianza Interpersonal, Latinobarómetro (2005).

En torno a confianza interpersonal y eficacia estatal para América Latina se obtiene una correlación estadística igual a 0,34 (ver Gráfico III) ${ }^{11}$.

Por su parte en lo que respecta a la correlación entre eficacia estatal y confianza institucional gubernamenta ${ }^{12}$ ésta es de 0,24 ; mientras que la co-variación entre la confianza institucional y la confianza interpersonal arroja un resultado más modesto del 0,14.

$\mathrm{Al}$ parecer las correlaciones no son fuertes ni concluyentes, pero, por el momento, al ser positivas, nos sirven para no descartar la hipótesis destacando que la relación directa entre eficacia estatal y confianza interpersonal resulta más fuerte que la que surgiría de mediar la confianza institucional. De este modo el primer argumento de la hipótesis 1) saldría reforzado con relación al segundo argumento de la misma. No obstante,

11. Si en vez de utilizar el subíndice A del ICG se utilizan por ejemplo indicadores de eficiencia como el Governance Effectiviness elaborado por el Banco Mundial (que mide a partir de encuestas las percepciones de calidad de servicios públicos, la calidad del servicio civil y el grado de independencia de las presiones políticas, la calidad en la formulación e implementación de políticas públicas y la credibilidad del gobierno para llevar adelante tales políticas) resulta que las correlaciones también son positivas $r=0,22$. Dicho esto la hipótesis gana fortaleza.

12. Bajo este ítem se promedia la confianza en las siguientes instituciones: Presidencia, Poder Judicial, Congreso, Policía, Gobierno Local, las Fuerzas Armadas y la Administración Pública. 
si se agrupan los 18 países bajo análisis tomando en consideración rangos de desempeño gubernamental/eficacia estatal, puede visualizarse a grandes rasgos como a mayor efectividad mayor nivel de confianza institucional y mayor nivel de capital social.

\section{GRÁFICO III}

CORRELACIÓN ENTRE CONFIANZA INTERPERSONAL Y EFICACIA ESTATAL (SUbÍNDICE DE REQUERIMIENTOS BÁSICOS-ICG)

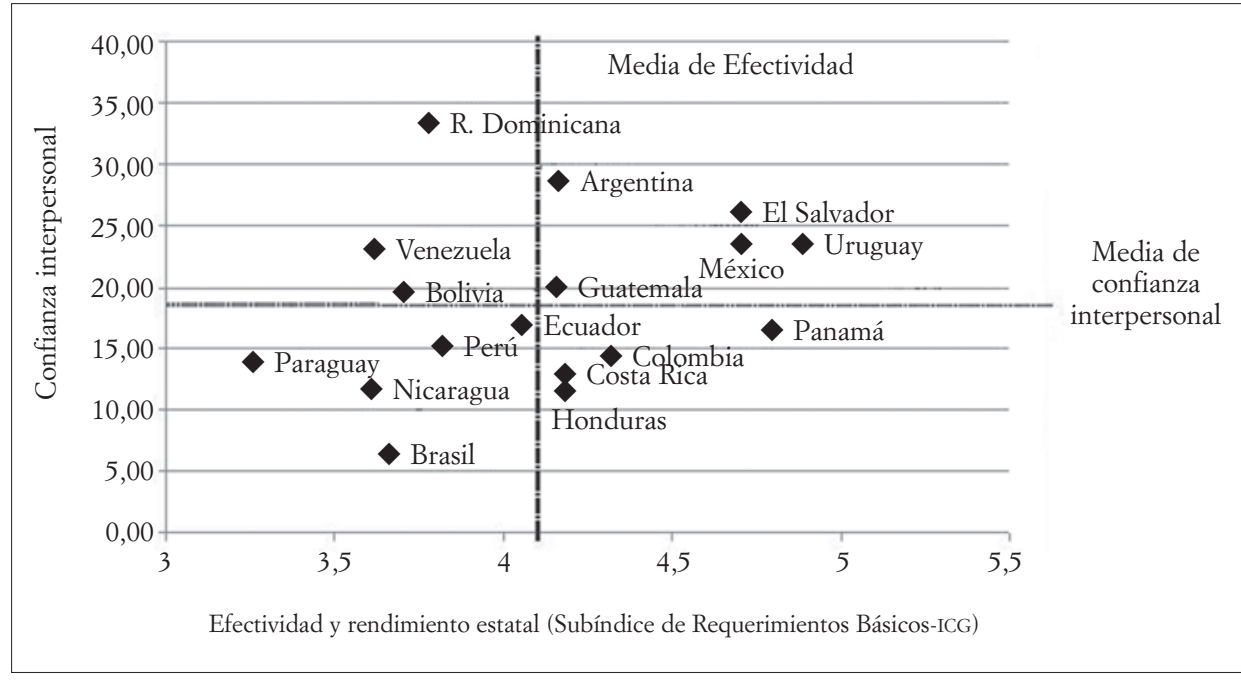

Fuente: Elaboración propia en base a datos de Latinobarómetro (2005) e ICG (2007-2008).

TABLA I

Niveles medios DE CONFIANZA INSTITUCIONAL GUBERNAMENTAL Y CONFIANZA INTERPERSONAL PARA LOS PAÍSES AGRUPADOS SEGÚN LOS NIVELES DE EFICACIA ESTATAL

\begin{tabular}{l|c|c|c}
\hline Valores medios en cada Grupo & $\begin{array}{c}\text { Países con Eficacia } \\
\text { Estatal ALTA } \\
\text { (media: } 4,58)\end{array}$ & $\begin{array}{c}\text { Países con Eficacia } \\
\text { Estatal MEDIA } \\
\text { (media: 4,09) }\end{array}$ & $\begin{array}{c}\text { Países con Eficacia } \\
\text { Estatal BAJA } \\
\text { (media: 3,73) }\end{array}$ \\
\hline $\begin{array}{l}\text { Confianza Institucional } \\
\text { Gubernamental }\end{array}$ & 12,12 & 10,96 & 9,93 \\
\hline Confianza Interpersonal & 20,83 & 21,37 & 14,75 \\
\hline
\end{tabular}

Fuente: Elaboración propia en base a datos de Latinobarómetro, 2005 e ICG (2007-2008). 
Por su parte, la segunda hipótesis propuesta sugería que, dado a que la igualdad económica y de oportunidades favorece la emergencia del capital social, las políticas estatales tendientes a mejorar la equidad son promotoras de capital social.

En lo que respecta a igualdad económica se ratifica en América Latina una correlación negativa entre confianza interpersonal y desigualdad económica (medida según coeficiente de Gini), cercana al $-0,40^{13}$. A tono con dicha afirmación pueden observarse casos extremos como el de Brasil, donde la peor distribución de la riqueza está acompañada por los peores niveles de capital social de la región; mientras que Uruguay, país que verifica la mayor igualdad económica de la región, sería a su vez el que mayores niveles de confianza interpersonal tiene.

En lo atinente a la igualdad de oportunidades, de los indicadores propuestos resulta que: el índice de capacidades básicas y el de educación superior y universitaria son los que más fuertemente se correlacionan con la confianza interpersonal $(\mathrm{r}=0,33$ para ambos casos), seguidos por las correlaciones verificadas entre confianza interpersonal y promedio de años de escolaridad $(r=0,32)$, la correlación con la tasa escolarización secundaria $(r=0,27)$ y, en último lugar, la correlación con la esperanza de vida al nacer $(\mathrm{r}=0,17)^{14}$.

Si se toma al gasto público social como medida de involucramiento y compromiso estatal en materia de equidad y se correlaciona con confianza interpersonal los resultados parecen más robustos $(\mathrm{r}=0,52)$, así también la correlación de la confianza con el gasto público social en educación $(r=0,34)$ y el gasto público en salud $(r=0,43)^{15}$.

En la Tabla II se observa entonces como el grupo de países con mayor gasto público social es a su vez el que tiene menores niveles de desigualdad, mayores niveles de confianza interpersonal y mayor eficacia estatal.

13. Para el Coeficiente de Gini Base de Datos Banco Mundial 2007, disponible en: http://hdrstats.undp.org/indicators/147.html.

14. Bases de datos utilizadas: para medir población con estudios universitarios y tasa de escolarización secundaria SITEAL, UNESCO y OEI (2006), disponible en: http://www.siteal.iipe-oei.org/. En el caso de estudios universitarios y escolarización secundaria las bases disponibles carecen de datos para Venezuela y República Dominicana, por tanto dichos países en este puntual caso fueron excluidos. Para medir el promedio de años de escolaridad se utilizaron datos del BM (2006) usados por A. SEN y B. KLiksBerg (2008: 130). Para medir esperanza de vida al nacer se utilizaron las bases de datos para los quinquenios 2005-2010: CELADE, Centro Latinoamericano y Caribeño de Demografía, División de Población de la CEPAL, disponible en http://www.cepal.org.

15. Tras reseñar en la primera parte de este trabajo los hallazgos encontrados en relación al papel de las políticas públicas de bienestar y el gasto social en la creación de condiciones más igualitarias y en los niveles de confianza social sería preciso poner de manifiesto los mecanismos explicativos en virtud de los cuales dichas variables se influyen y afectan. Por razones de espacios dicha tarea no se acomete en este artículo. Algunos avances al respecto pueden verse en M. C. GÜEMES (2011a) y M. C. GÜEMES (2011b). 
TABLA II

Niveles de Desigualdad, Confianza Interpersonal y Eficacia Estatal para PAÍSES AGRUPADOS EN FUNCIÓN DE LOS NIVELES DE GASTO PÚBLICO SOCIAL PER CÁPITA

\begin{tabular}{l|c|c|c}
\hline Valores medios en cada Grupo & $\begin{array}{c}\text { Países con gasto } \\
\text { público social } \\
\text { ALTO } \\
\text { (media: } 997,2)\end{array}$ & $\begin{array}{c}\text { Países con gasto } \\
\text { público social } \\
\text { MEDIO } \\
\text { (media: 336,6) }\end{array}$ & $\begin{array}{c}\text { Países con gasto } \\
\text { público social } \\
\text { BAJO } \\
\text { (media: } 125,8)\end{array}$ \\
\hline Desigualdad Económica & 50,66 & 53,46 & 54,01 \\
\hline Confianza Interpersonal & 21,25 & 19,16 & 15,81 \\
\hline Eficacia Estatal & 4,38 & 4,2 & 3,87 \\
\hline
\end{tabular}

Fuente: Elaboración propia con datos de ICG (2007-2008), Latinobarómetro (2005) y CEPAL (2005 y 2006).

\section{III.3. Recapitulación sobre los resultados obtenidos en América Latina}

a. La región latinoamericana posee una media de capital social baja en referencia a otras regiones, así como una distribución muy desigual del capital social entre los países que la integran ${ }^{16}$.

b. Los niveles de calidad democrática y de desarrollo humano pueden estar acompañados de altos niveles de capital social. Sin embargo, existen casos como el de Brasil, donde los niveles de desarrollo y calidad democrática son relativamente altos y sin embargo son bajísimos los niveles de capital social, o el de República Dominicana, donde los niveles de confianza interpersonal son relativamente altos mientras sus niveles de desarrollo y calidad democrática son más bien bajos.

c. En cuanto a la primera hipótesis propuesta, se verifica una correlación positiva entre confianza interpersonal y efectividad gubernamental $(r=0,34)$, mas el Gráfico III ayuda a comprender que difícilmente puede hablarse de un comportamiento lineal o regular entre ambas variables. Dicho lo cual, se intuye la presencia de alguna otra variable que pueda estar condicionando tanto la eficacia estatal como el capital social. En este sentido, y como más tarde se mencionará, las investigaciones que proponen las configuraciones y estructuras histórico-nacionales como variable explicativa (o al menos relevante a los efectos causales) tanto de la eficacia y el desempeño estatal como de las relaciones sociales parecen muy sugerentes al respecto reclamando estudios en profundidad que van más allá de los análisis cuantitativos.

d. En lo que respecta a la segunda hipótesis, también encontramos correlaciones favorables entre:

16. La heterogeneidad al interior de la región no es exclusiva de América Latina, como puede verse en la tabla del Anexo, también al interior de Europa, Asia y demás regiones pueden vislumbrase heterogeneidades. 
i. Igualdad económica ${ }^{17} \mathrm{y}$ de oportunidades y confianza interpersonal (correlación negativa con desigualdad económica de $-0,4$ y correlaciones positivas cercanas al 0,33 en indicadores educativos varios).

ii. Gasto público y confianza interpersonal $(0,52)$.

Estos últimos resultados conducen a reflexionar la ligazón existente entre desarrollo de los sistemas de bienestar (y el involucramiento del Estado a tal efecto) y niveles de capital social. En este sentido podría señalarse una cierta coincidencia entre aquellos países que como Argentina y Uruguay desarrollaron sistemas de bienestar universales y poseen así mismo los más altos niveles de capital social y, por otro lado, aquellos países signados por profundas desigualdades sociales donde los esquemas de bienestar han sido prácticamente inexistentes o sumamente informales como pueden ser Nicaragua, Honduras o Paraguay, y que a su vez cuentan con los peores niveles de capital social. No obstante, tal asociación es a primera vista un poco apresurada ya que también existen países con sistemas de bienestar sumamente informales como Guatemala y otros con niveles intermedios de capital social o países como Chile, que han atravesado procesos de modernización económica y cuentan con regímenes de bienestar «productivistas» y sin embargo sus niveles de confianza social son de los más bajos en la región. Atento a ello sería preciso cumplimentar tales observaciones indagando en los modelos, formas y evolución de los regímenes de Bienestar en América Latina así como en la distribución social del bienestar y en el aprovechamiento de dicho bienestar por los diferentes grupos sociales (Martínez 2006; Espina 2008; Katzman 2007; Rothstein 2008; Carrera y Muñoz Bustillo 2009) ${ }^{18}$.

\section{CONCLUSIONES}

A pesar de ser un concepto tan polisémico, con notables debilidades y vacíos, se decidió abordar el capital social pues se entiende que posee la ventaja de revalorizar bienes sociales intangibles como la confianza o la reciprocidad en el marco de la problematización del desarrollo económico.

Se volvían a emparentar las preocupaciones de la sociología clásica con las preocupaciones de los pioneros del desarrollo y se rechazaba un abordaje exclusivamente economicista a situaciones complejas. En términos más específicos, a la par que se

17. No obstante, se ha señalado que la relación entre la distribución del capital social y la del ingreso no es lineal ni proporcional resultando la disponibilidad de capital social en los grupos más pobres variable según se toma en cuenta la capacidad de movilización de estos grupos o su dotación de recursos asociativos (R. ATRIA 2003).

18. Otra cuestión importante a señalar es que los Estados de Bienestar dependen en buena medida de la economía y de los recursos que el Estado pueda recaudar, por tanto, aquellos países que no han logrado sostener el crecimiento y productividad económica difícilmente puedan proveer políticas de bienestar universales (B. RothSTEIn y E. UsLANER, 2005). Para una lectura más optimista véase (A. SEN y B. KLIKSBERg 2008). 
intentaba examinar qué factores extraeconómicos se ocultan detrás de experiencias de desarrollo exitosas, se reivindicaba el papel de las relaciones sociales y se reincorporaban factores políticos-estructurales en el debate.

Así, los primeros estudios comparados que incorporaban la dimensión del capital social verificaban la presencia de regiones bendecidas con altos niveles de stocks de capital social, humano y económico, contrapuestas a territorios entrampados en «círculos viciosos». Dicha observación condujo a la necesidad de problematizar la creación del capital social.

Desde una visión política constructivista del capital social, y centrando la atención en la realidad de América Latina, se postuló al Estado como un actor capaz de romper tales círculos viciosos y promover el capital social. Al efecto, se presentaron dos hipótesis tendientes a explorar en qué medida el eficaz desempeño de un Estado, así como su involucramiento político en el área de bienestar social, se correlacionaba con el capital social.

Una primera y modesta aproximación cuantitativa a tales hipótesis, si bien no las confirma categóricamente, al menos, sugiere no rechazarlas e invita a cumplimentar tal estudio con un abordaje cuantitativo más complejo así como con un estudio en profundidad que presten mayor atención a:

1) Las configuraciones históricas y estructurales en las que se inserta y contextualiza la confianza social, esto es: la arquitectura sobre la que descansan las relaciones sociales y el modo en que las políticas públicas e intervenciones estatales han moldeado las mimas (Portes y Hoffman 2003; Basualdo y Arceo 2006; Fernández, Güemes y Vigil 2006).

2) El modo en que el Estado construye o debilita las estructuras de socialización y espacios de interacción (como la escuela o el barrio) en los cuales se desarrolla y gesta el capital social (Güell 2002; Katzman 2003, 2007; De Maza 2001).

3) La influencia y efecto que el modelo político «privatizador y mercantilizador» de la vida humana, aplicado a la mayoría de los países latinoamericanos en las últimas décadas, tuvo sobre la distribución espacial y social del capital social, las macroestructuras sociales, políticas y económicas, los espacios de interacción social y los imaginarios y sentidos de pertenencia social (Lechner 2000; Katzman 2003; Portes y Hoffman 2003).

Todo ello lleva a concluir que liberar los focos de energía social subyacentes en América Latina (Hirschman 1986), que tan elementales son al desarrollo económico, demanda mucho más que la implementación de políticas bienintencionadas de fomento de la cooperación basadas en palos y zanahorias, estrategias microlocales de reactivación del sentimiento comunitario o políticas de educación cívica. Es preciso fortalecer la reflexión, contextualizar y readecuar herramientas teóricas como las analizadas a contextos específicos como el latinoamericano e insertar dichas claves analíticas en una estrategia más general que se plantee alterar las bases estructurales de la desigualdad en la región volviendo a integrar a la ciudadanía en un proyecto común y compartido. 
MARÍA CECILIA GÜEMES

ESTADO Y CAPITAL SOCIAL EN AMÉRICA LATINA

\section{BibliografíA}

Aguiar, Fernando. Lógica de la cooperación. Zona Abierta, 1990, vol. 54/55: 7-42.

Aguilar Villanueva, Luis. El aporte de la Política Pública y de Nueva Gestión Pública a la Gobernanza. Revista del CLAD Reforma y Democracia, 2007, vol. 39.

Almond, Gabriel y Verba, Sidney. La Cultura Cívica. Estudios sobre la participación política democrática en cinco naciones. Madrid: Editorial Euramérica, 1970.

ATRIA, Raúl. Capital social: concepto, dimensiones y estrategias para su desarrollo. En ATRIA, Raúl. Capital social y reducción de la pobreza en América Latina y el Caribe: en busca de un nuevo paradigma. Santiago de Chile: CEPAL, 2003.

AYERBE, Miguel et al. El capital social de las organizaciones y su entorno: conceptualización teórica, medición e intervención en la generación del capital social. EKONOMIAZ. Revista Vasca de Economía, 2005, vol. 59: 14-47.

BAgNASCO, Arnaldo. Teoría del capital social y political economy comparada. En BAGNASCO, Arnaldo et al. El capital Social. Instrucciones de uso. Buenos Aires: FCE, 2003 (89-122).

BASUALDO, Eduardo y ARCEO, Enrique (comps.). Neoliberalismo y sectores dominantes. Tendencias globales y experiencias nacionales. Buenos Aires: Ed. CLACSO, 2006.

BEBBINGTON, Anthony. Estrategias de vida y estrategias de intervención: el capital social y los programas de superación de la pobreza. En ARRIAGA, Irma. Aprender de la experiencia. El capital social en la superación de la pobreza. Santiago de Chile: CEPAL, Publicación de Naciones Unidas, 2005.

Borx, Carles y Postner, Daniel. Capital Social y Democracia. Revista Española de Ciencia Política, 2000, vol. 1 (2): 159-185.

BouRdIEU, Pierre. El capital social. Apuntes provisionales. Zona Abierta, 2001, vol. 94/95: 83-87.

BOWLES, Samuel y GINTIS, Herbert. Social Capital and Community Governance. Economic Journal, 2002, vol. 102 (483): 419-436.

Caballero, Gonzalo y Kingston, Christopher. Capital social e instituciones en el proceso de cambio económico. EKONOMIAZ. Revista Vasca de Economía, 2005, vol. 59: 70-91.

CARRERA, Miguel y MuÑoz DE Bustillo, Rafael. El reto de la pobreza y la desigualdad en América Latina. Revista Sistema, 2009, vol. 208-209: 73-96.

CEPAL. Agenda social capital social: sus potencialidades y limitaciones para la puesta en marcha de políticas y programas sociales. Panorama social de América Latina 2001-2002. CEPAL. Disponible en: http://www.cepal.org/cgi-bin/getProd.asp?xml=/publicaciones/xml/4/11254/ P11254.xml\&xsl=/dds/tpl/p9f.xsl\&base=/tpl/top-bottom.xslt.

Coleman, James. Capital Social y creación de capital humano. Zona Abierta, 2001, vol. 94/95: 47-81.

Cornejo, Celia. Capital social, competitividad. EKONOMIAZ. Revista Vasca de Economía, 2005, vol. 59: 93-117.

Dasgupta, Partha. Social Capital and Economic Performance: Analytics. En Ostrom, Elinor y AHN, Toh-Kyeong (eds.). Critical Studies in Economic Institutions: Foundations of Social Capital. Cheltenham: Edward Elgar, 2003 (309-339).

De LA MAZA, Gonzalo. Sociedad Civil y Construcción de Capital Social en América Latina: ¿Hacia dónde va la investigación? Polis. Revista de la Universidad Bolivariana, 2001, vol. 1 (002). Disponible en http://www.revistapolis.cl/2/maza.pdf.

Della PORTA, Donatella. Social Capital, Belief in Goverment, and Political Corruption. En PHARR, Susan y PuTnam, Robert. Disaffected Democracies. Wath's Troubling the Trilateral Countries. New Jersey: Princeton University Press, 2000 (202-230). 
DíAZ AlBERTINI, Javier. Capital Social, Organizaciones de Base y el Estado: Recuperando los eslabones perdidos de la sociabilidad. Ponencia presentada para la Conferencia Regional sobre capital social y reducción de la pobreza en América Latina y el Caribe. Santiago de Chile: CEPAL, septiembre de 2001.

Durlauf, Steven N. On the Empirics of Social Capital. The Economic Journal, 2002, vol. 112 (483): 459-479.

DurSTON, John. Construyendo capital social comunitario. Revista de la CEPAL, 1999, vol. 69: 103 118.

DURSTON, John. ¿Qué es el capital social comunitario? Serie de Políticas Sociales CEPAL, n. ${ }^{\circ}$ 38, 2000.

DURSTON, John. El capital social en seis comunidades campesinas de Chile: adelantos y desafíos de una investigación en marcha. En DURSTON, John y MiRANDA, Francisca (comps.). Capital social y políticas públicas en Chile. Investigaciones recientes. Santiago de Chile: CEPAL, Publicación de Naciones Unidas, vol. I, 2003 (39-52).

ELSTER, Jon. Racionalidad, moralidad y acción colectiva. Zona Abierta, 1990, vol. 54-55: 43-67.

ELSTER, Jon. Tuercas y Tornillos. Una introducción a los conceptos básicos de las ciencias sociales. Barcelona: Ed. Gedisa, 2003.

EsPINA, Álvaro. Modernización, estadios de desarrollo económico y regimenes de bienestar en América Latina. Fundación Carolina, 2008, Documento de Trabajo, n. ${ }^{\circ} 28$. Disponible en http://www.fundacioncarolina.es/es-ES/publicaciones/documentostrabajo/Paginas/Documentosdetrabajo.aspx.

Evans, Peter. El Estado como problema y como solución. Desarrollo Económico, 1996a, vol. 35 (140): 529-562.

Evans, Peter. Government Action, Social Capital and Development: Reviewing the Evidence on Synergy. World Development, 1996b, vol. 24 (6): 1119-1132.

FERNÁNDEZ, Víctor R.; GÜEMES, Cecilia y VigIL, José. Estado y Desarrollo en los discursos del Banco Mundial. Revista Problemas del Desarrollo-UNAM, 2006, vol. 37 (144): 33-72.

GranovetTer, Mark. Economic Action and Social Structure: The Problem of Embeddedness. The American Journal of Sociology, 1985, vol. 91 (3): 481-510.

GroOtAER, Christiaan y VAN BASTELAER, Thierry. Understanding and Measuring Social Capital: A Synthesis of Findings and Recommendations from the Social Capital Inititative. Work Bank. Social Capital Inititative, 2001, Working Paper, n. ${ }^{\circ}$ 24. Disponible en: http://siteresources.worldbank.org/INTSOCIALCAPITAL/Resources/Social-Capital-Initiative-WorkingPaper-Series/SCI-WPS-24.pdf.

GÜELL, Pedro. ¿Quién le apuesta al Capital Social en América Latina? Conferencia pronunciada en Universidad del Rosario. Bogotá, Colombia, octubre, 2002.

GÜEMES, M. ${ }^{a}$ Cecilia. Estrategia metodológica para un análisis del impacto de las políticas públicas sobre la confianza social. Documento Borrador a publicarse próximamente en Obra colectiva, 2011a.

GÜEMES, M. ${ }^{a}$ Cecilia. Políticas de bienestar y confianza social. El impacto de las reformas neoliberales en Argentina. Documento Borrador a publicarse próximamente en Obra colectiva, 2011b.

GÜEMES, M. ${ }^{a}$ Cecilia. Estrategia metodológica para un análisis del impacto de las políticas públicas sobre la confianza social. Revista Circunstancia. Instituto Universitario Ortega y Gasset (en prensa).

GÜemes, M. ${ }^{a}$ Cecilia; Fernández, Víctor R. y Magnin, Juan Pablo. Estado y Capital Social, las nuevas «Keywords» del Desarrollo. Trabajo presentado en el I Encuentro Nacional de Ciencias Sociales «Las Ciencias Sociales frente a los desafíos de América Latina en el siglo XXI». Villa María, Córdoba, Argentina, 1 y 2 de noviembre de 2006. 
Hall, Peter. El capital social en Gran Bretaña. En Putnam, Robert. El declive del capital social. Barcelona: Ed. Galaxia Gutenberg, 2002 (35-92).

HerReros, Francisco. Republicanismo, Capital Social y Democracia. Revista de Estudios Políticos (Nueva Época), 2002a, vol. 117: 293-312.

HERREROS, Francisco. ¿Son las relaciones sociales una fuente de recursos? Una definición de capital social. Papers. Revista de Sociología, 2002b, vol. 67: 129-148.

Herreros, Francisco. Confianza y cooperación en ausencia de Estado. Revista Internacional de Sociología (RIS), 2007, vol. LXV (46): 161-183.

HerReros, Francisco y CRIADO, Henar. El problema de la formación del capital social. Estado, asociaciones voluntarias y confianza generalizada. Zona Abierta, 2001, vol. 94/95: 201-231.

HerREROS, Francisco y CRIADO, Henar. The State and the Development of social trust. International Political Sciencie Review, 2008, vol. 29 (1): 53-71.

HerReros, Francisco y De Francisco, Andrés. Introducción: el capital social como programa de investigación. Zona Abierta, 2001, vol. 94/95: 1-46.

HiRSCHMAN, Albert. El avance en colectividad. Experimentos populares en la América Latina. MéxiCO DF: FCE, 1986a.

HIRSCHMAN, Albert. Interés privado y acción pública. Mexico, DF: FCE, 1986b.

IngleHART, Ronald. The renaissance of Political Culture. The American Political Science Review, 1988, vol. 82 (4): 1203-1230.

JORDANA, Jacint. Instituciones y capital social: ¿qué explica qué? Revista Española de Ciencia Política, 2000, vol. 1 (2): 187-210.

KANHEMAN, Daniel. Mapas de racionalidad limitada: psicología para una economía conductual. RAE. Revista Asturiana de Economía, 2003, vol. 28: 181-225.

Katzman, Rubén. Capital social y sociedad civil en América Latina. Presentación en el Seminario BID sobre la Agenda Ética Pendiente en América Latina. Universidad de la República, Montevideo, Uruguay, 18 y 19 de diciembre de 2003.

KATZMAN, Rubén. La calidad de las relaciones sociales en las grandes ciudades de América Latina: viejos y nuevos determinantes. Revista Pensamientos Iberoamericanos, 2007, vol. 1: 177-205.

KaWACHI, Ichiro; KenNedy, Bruce; Lochner, Kimberly y Prothrow, Deborah. Social Capital, Income Inequality and Mortality. American Journal of Public Health, 1997, vol. 87 (I.9): 14911498.

Kliksberg, Bernardo. Capital social y cultura. Claves olvidadas del desarrollo. Documento de Divulgación 7. Banco Interamericano de Desarrollo. Instituto para la Integración de América Latina y el Caribe -INTAL-, 2000 Disponible en: http://www.iadb.org/Intal/aplicaciones/uploads/publicaciones/e_INTAL_DD_07_2000_kliksberg.pdf.

KNACK, Stephen. Trust, Associational Life and Economic Performance. Paper presented at the HRDCOECD International Symposium on The Contribution of Investment in Human and Social Capital to Sustained Economic Growth and Well Being, April, 2000.

KnACK, Stephen y KeEFER, Philip. Does Social Capital Have an Economic Payoff? A Cross-Country Investigation. The Quarterly Journal of Economics, 1997, vol. 112 (4): 1251-1288.

LECHNER, Norbert. Desafíos de un desarrollo humano: individualización y capital social. Instituciones y Desarrollo, 2000, vol. 7. Disponible en: http://www.iigov.org/id/attachment.drt?art= 187652.

Levi, Margaret. A State of Trust. En BraithWAite, Valerie y Levi, Margaret (eds.). Trust and Governance. Nueva York: Ed. Russell Sag, 1998.

LEVI, Margaret. Capital social y asocial: ensayo crítico sobre Making Democracy Work. Zona Abierta, 2001, vol. 94-95: 105-120. 
Levine, Daniel y MolinA, José. La calidad de la democracia en América Latina. América Latina Hoy, 2007, vol. 45: 17-46.

LIN, Nan. Building a Theory of Social Capital. Connection, 1999, vol. 22 (1): 28-51.

LINARES, Francisco. El problema de la emergencia de normas sociales en la acción colectiva. Una aproximación analítica. Revista Internacional de Sociología, 2007, vol. LXV (46): 131-160.

LIPSEP, Seymour Martin. Some Social Requisites of Democracy: Economic Development and Political Legitimacy. The American Political Science Review, 1959, vol. 53 (1): 69-105.

Martínez Franzoni, Juliana. Regímenes de bienestar en América Latina. Fundación Carolina y CEALCI, Avance de Investigación, 2006. Disponible en: http://www.fundacioncarolina.es/esES/publicaciones/avancesinvestigacion/Documents/regimenesdebienestarenamericalatina.pdf.

MotA, Fabiola y SubIRATs, Joan. El quinto elemento: el capital social de las comunidades autónomas. Su impacto sobre el funcionamiento del sistema político autonómico. Revista Española de Ciencia Política, 2000, vol. 1 (2): 123-158.

MотA DíAZ, Laura. El capital social: un paradigma en el actual debate sobre el desarrollo. Tendencias y problemas. Revista Espiral. Estudios sobre Estado y Sociedad, 2002, vol. IX (25): 37 65.

NewTon, Kenneth y NorRIs, Pippa. Confidence in Public Institutions: Faith, Culture or Performance? En Pharr, Susan y PuTnam, Robert. Disaffected Democracies. Wath's Troubling the Trilateral Countries. New Jersey: Princeton University Press, 2000 (52-73).

OfFE, Claus y Fuch, Susanne. ¿Se halla en declive el capital social? El caso alemán. En PutNAm, Robert. El declive del capital social. Barcelona: Ed. Galaxia Gutenberg, 2002 (345-425).

OlazARAN, Mikel; LAVÍA, Cristina y OTERO, Beatriz. Cooperación, conocimiento e innovación: políticas y agentes regionales de I+D. EKONOMIAZ. Revista Vasca de Economía, 2005, vol. 59: 186-213.

Olson, Mancur. La lógica de la acción colectiva. En BATTLE, Albert (ed.). Diez textos básicos de ciencia política. Barcelona: Ariel, 1992 (203-219).

Ostrom, Elinor y AHN, Toh-Kyeong. Una perspectiva del capital social desde las ciencias sociales: capital social y acción colectiva. Revista Mexicana de Sociología, vol. 1, 2003: 155-233.

PARAmio, Ludolfo. Decisión racional y acción colectiva. Leviatan, vol. 79, 2000: 65-83.

PISELLI, Fortunata. Capital social: un concepto situacional y dinámico. En BAGNASCO, Arnaldo et al. El capital Social. Instrucciones de uso. Buenos Aires: FCE, 2003 (53-88).

Pizzorno, Alessandro. Por qué pagamos la nafta. Por una teoría del capital social. En BAGNASCO, Arnaldo et al. El capital Social. Instrucciones de uso. Buenos Aires: FCE, 2003 (19-52).

PORTES, Alejandro. Social Capital: Its Origins and Applications in Modern Sociology. Annual Review of Sociology, 1998, vol. 24: 1-24.

PORTES, Alejandro y Hoffman, Kelly. La estructura de clases en América Latina: Composición y cambios durante la era Neoliberal. Desarrollo Económico, 2003, vol. 43 (171): 355-385.

PORTES, Alejandro y LANDOlT, Patricia. Social Capital: Promise and Pitfalls of Its Role in Development. Journal of Latin American Studies, 2000, vol. 32 (2): 529-547.

Putnam, Robert. Making Democracy Work. Civic traditions in Modern Italy. Princeton: Princeton University Press, 1993.

Putnam, Robert. La comunidad próspera. El capital social y la vida pública. Zona Abierta, 2001, vol. 94-95: 89-104.

Putnam, Robert y Goss, Kristin. Introducción. En Putnam, Robert. El declive del capital social. Barcelona: Ed. Galaxia Gutenberg, 2002 (7-33).

RodrígueZ, Paula y RomÁn DEL Río, Carlos. El capital social como factor de competitividad y desarrollo empresarial. EKONOMIAZ. Revista Vasca de Economia, 2005, vol. 59: 214-231. 
RoIz, Javier. La recuperación del buen juicio. Madrid: Editorial Foro Interno, 2003.

RothSTEIn, Bo. Capital social, socialdemocracia y neocorporativismo. En PUTNAM, Robert. El declive del capital social. Barcelona: Ed. Galaxia Gutenberg, 2002 (94-164).

RothsteIn, Bo. Is the Universal Welfare State a Cause or an Effect of Social Capital? QoG Working Paper Series, 2008: 16.

Rothstein, Bo y Uslaner, Eric. All for All. Equality, Corruption, and Social Trust. World Politics, 2005, vol. 58: 41-72.

Sabatini, Fabio. Social Capital, Public Spending and the Quality of Economic Development. Presentation at the COE/JEPA (Center of Excellence/Japan Economic Policy Association) Joint International Conference, 'Towards a New Economic Paradigm'. Kobe, Japan, 17-18 December 2005.

SÁnCHEZ, José y PenA, José. Actividad asociativa, confianza y generación de capital social: evidencia empírica. EKONOMIAZ. Revista Vasca de Economía, vol. 2 (59), 2005: 136-159.

ScHOLZ, John y GraY, Wayne. Can Government Facilitate Cooperation? An Informational Model of OSHA Enforcement. American Journal of Political Science, 1997, vol. 41 (3): 693-717.

SEN, Amartya. Los tontos racionales. Una crítica de los fundamentos conductistas de la teoría económica. En HAHN, Frank y Hollis, Martin (eds.). Filosofía y teoría económica. México: FCE, 1986 (172-217).

Sen, Amartya y KLIKSBERG, Bernardo. Primero la gente. Barcelona: Ed. Deusto, 2008.

SIDES, John. It takes two: The reciprocal relationship between Social Capital and Democracy. Working Paper Institute of Governmental Studies. University of California, Berkeley, 1999.

STOLLE, Dietlind. Jugando a los bolos, jugando solos: el desarrollo de confianza generalizada en las asociaciones voluntarias. Zona Abierta, 2001, vol. 94-95: 161-199.

TARROW, Sidney. Making social science work across space and time: A critical reflection on Robert Putnam's Making Democracy Work. The American Political Sciencie Review, 1996, vol. 2 (90): 389-397.

TAYLOR, Michael. El Buen Gobierno: sobre la jerarquía, el capital social y las limitaciones de la teoría de la elección racional. Zona Abierta, 2001, vol. 94/95: 121-160.

TorCAL, Mariano y MonTERo, José Ramón. La formación y consecuencias del capital social en España. Revista Española de Ciencia Política, 2000, vol. 1 (2): 79-121.

Trigilia, Carlos. Capital social y desarrollo local. En BAGNASCO, Arnaldo et al. El capital Social. Instrucciones de uso. Buenos Aires: FCE, 2003 (123-155).

Weber, Max. La ética protestante y el espíritu del capitalismo. Madrid: Itsmos, 1998.

WoOlCOCK, Michael y NARAYAN, Deepa. Capital Social: implicaciones para la teoría, la investigación y las políticas sobre el desarrollo. Banco Mundial, Research Observer, 2000, vol. 15 (2): $225-249$.

Wuthnow, Robert. El carácter cambiante del capital social en Estados Unidos. En PutnAm, Robert. El declive del capital social. Barcelona: Ed. Galaxia Gutenberg, 2002 (543-620). 
VI. ANEXO

VI.1. Comparación entre niveles capital social de América Latina y otras regiones

TABLA I

CONFIANZA INTERPERSONAL POR REGIONES. AÑOS 2005-2007

\begin{tabular}{|c|c|}
\hline Confianza interpersonal & $\%$ de se puede confiar \\
\hline Países nórdicos & 57,3 \\
\hline Holanda & 45 \\
\hline Suecia & 68 \\
\hline Finlandia & 58,9 \\
\hline EE.UU. y excolonias británicas & 44,85 \\
\hline EE.UU. & 39,3 \\
\hline Canadá & 42,8 \\
\hline Australia & 46,1 \\
\hline Nueva Zelanda & 51,2 \\
\hline Asia & 37,06 \\
\hline Japón & 39,1 \\
\hline Corea del Sur & 28,2 \\
\hline China & 52,3 \\
\hline Taiwán & 24,2 \\
\hline Tailandia & 41,5 \\
\hline Europa & 29,74 \\
\hline Francia & 18,8 \\
\hline Gran Bretaña & 30,5 \\
\hline Italia & 29,2 \\
\hline España & 20 \\
\hline Polonia & 19 \\
\hline Alemania & 36,8 \\
\hline Suiza & 53,9 \\
\hline América Latina (Promedio) & 12,67 \\
\hline México & 15,6 \\
\hline Colombia & 14,5 \\
\hline Argentina & 17,6 \\
\hline Brasil & 9,4 \\
\hline Chile & 12,6 \\
\hline Perú & 6,3 \\
\hline
\end{tabular}

Fuente: World Value Survey (2005-2008). 


\section{VI.2. Comentarios respecto a los indicadores de capital social}

Atento a los estudios empíricos realizados por otros autores se pueden diferenciar 7 indicadores de capital social: 1) Confianza interpersonal, 2) Trabajo comunitario, 3) Asociacionismo formal, 4) Asociacionismo informal (participado en manifestaciones autorizadas o firma de peticiones), 5) Atención al entorno (asiduidad con que se consultan periódicos, radio y TV), 6) Grado de Satisfacción con la vida y 7) Expectativas de futuro mejor para sus hijos.

TABLA II

CORRELACIONES ENTRE LOS DIFERENTES COMPONENTES DEL CAPITAL SOCIAL

\begin{tabular}{l|c|c|c|c|c|c|c}
\hline Correlaciones & $\begin{array}{c}\text { Confianza } \\
\text { interper- } \\
\text { sonal }\end{array}$ & $\begin{array}{c}\text { Trabajo } \\
\text { comuni- } \\
\text { tario }\end{array}$ & $\begin{array}{c}\text { Asocia- } \\
\text { cionismo }\end{array}$ & $\begin{array}{c}\text { Asocia- } \\
\text { cionismo } \\
\text { informal }\end{array}$ & Atención & $\begin{array}{c}\text { Satisfac- } \\
\text { ción con } \\
\text { la vida }\end{array}$ & $\begin{array}{c}\text { Expecta- } \\
\text { tivas de } \\
\text { futuro }\end{array}$ \\
\hline $\begin{array}{l}\text { Confianza } \\
\text { interpersonal }\end{array}$ & 1,00 & & & & & & \\
\hline $\begin{array}{l}\text { Trabajo } \\
\text { comunitario }\end{array}$ & 0,18 & 1,00 & & & & & \\
\hline Asociacionismo & 0,45 & 0,41 & 1,00 & & & & \\
\hline $\begin{array}{l}\text { Asociación } \\
\text { informal }\end{array}$ & 0,38 & 0,49 & 0,29 & 1,00 & & & \\
\hline Atención & 0,63 & 0,56 & 0,80 & 0,51 & 1,00 & & \\
\hline $\begin{array}{l}\text { Satisfación } \\
\text { con la vida }\end{array}$ & 0,28 & 0,02 & $-0,28$ & 0,05 & $-0,15$ & 1,00 & \\
\hline $\begin{array}{l}\text { Expectativas } \\
\text { futuro }\end{array}$ & $-0,22$ & 0,17 & 0,36 & $-0,16$ & 0,19 & $-0,53$ & 1,00 \\
\hline
\end{tabular}

Fuente: Elaboración propia en base a datos de Latinobarómetro (2005).

La diferencial vinculación entre los indicadores propuestos es altamente problemática. En primer término se entremezclan indicadores que evalúan opiniones con aquellos otros que miden cantidades de asociaciones en las que se participa. En segundo lugar en aquellos casos donde se encuentran fuertes correlaciones no se puede conocer con precisión el tipo de relación existente, si ambos indicadores miden lo mismo o si atienden aspectos diferentes del fenómeno en estudio o si uno condiciona o causa al otro. En tercer y último lugar resulta que ciertos países son fuertes en algunos de dichos indicadores y no en otros. En torno a estas razones es por lo cual preferimos atenernos, pese a sus debilidades, al indicador más utilizado en los estudios comparados: la confianza interpersonal. En el gráfico que sigue puede observarse el parecido comportamiento entre confianza interpersonal y un posible índice agregado promediando los indicadores antes propuestos. 


\section{GRÁFICO I}

CONFIANZA INTERPERSONAL E ÍNDICE AGREGADO DEL CAPITAL SOCIAL. AÑo 2005

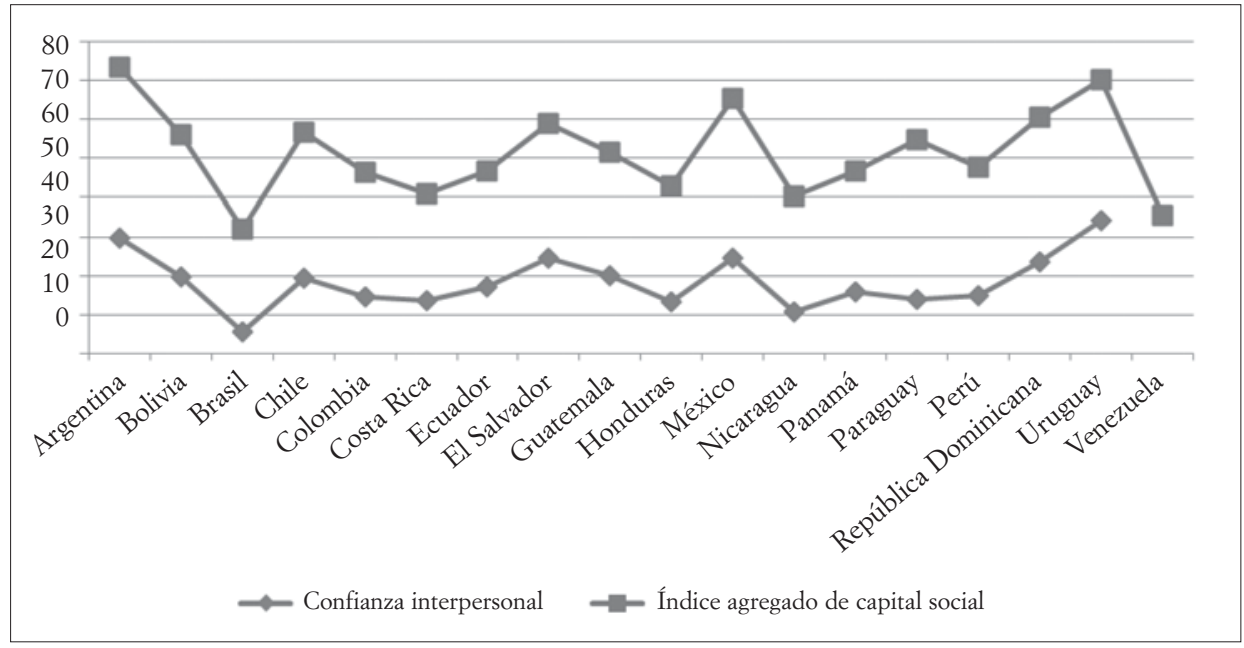

Fuente: Elaboración propia con base de datos Latinobarómetro (2005). 